\title{
pombalina
}

(8)

\section{Aires Barbosa e l'esperienza poetica dell'umanesimo italiano}

Autor(es): Pantani, Italo

Publicado por: Imprensa da Universidade de Coimbra; Sapienza. Universitá di Roma

URL

persistente: URI:http://hdl.handle.net/10316.2/38769

DOI: $\quad$ DOI:http://dx.doi.org/10.14195/978-989-721-044-0_2

Accessed : $\quad$ 26-Apr-2023 12:23:17

A navegação consulta e descarregamento dos títulos inseridos nas Bibliotecas Digitais UC Digitalis, UC Pombalina e UC Impactum, pressupõem a aceitação plena e sem reservas dos Termos e Condições de Uso destas Bibliotecas Digitais, disponíveis em https://digitalis.uc.pt/pt-pt/termos.

Conforme exposto nos referidos Termos e Condições de Uso, o descarregamento de títulos de acesso restrito requer uma licença válida de autorização devendo o utilizador aceder ao(s) documento(s) a partir de um endereço de IP da instituição detentora da supramencionada licença.

Ao utilizador é apenas permitido o descarregamento para uso pessoal, pelo que o emprego do(s) título(s) descarregado(s) para outro fim, designadamente comercial, carece de autorização do respetivo autor ou editor da obra.

Na medida em que todas as obras da UC Digitalis se encontram protegidas pelo Código do Direito de Autor e Direitos Conexos e demais legislação aplicável, toda a cópia, parcial ou total, deste documento, nos casos em que é legalmente admitida, deverá conter ou fazer-se acompanhar por este aviso. 


\section{Aires Barbosa na}

\section{Cosmopólis Renascentista}

Italo Pantani, Margarida Miranda \& Henrique Manso (coordenadores)

IMPRENSA DA UNIVERSIDADE DE COIMBRA

SAPIENZA. UNIVERSITÁ DI ROMA 


\section{Aires Barbosa \\ E L'ESPERIENZA POETICA DELL'UMANESIMO ITALIANO}

Italo Pantani

“La Sapienza”, Università di Roma

Due evidenti, e già note costanti è dato rinvenire nella prestigiosa attività culturale di Aires Barbosa, tanto nei ventotto anni dell'insegnamento nell'Università di Salamanca (1495-1523), quanto nei sette trascorsi presso la corte reale portoghese come precettore del giovanissimo cardinale Alfonso (1523-1530), quanto infine negli ultimi dieci passati nel ritiro del suo paese natale (1530-1540): una coerente dedizione ai principi della dottrina cattolica, e la centralità pressoché assoluta assegnata alla poesia, tanto come materia di studio e insegnamento, quanto come pratica creativa. Lo comprova la massima parte delle opere da lui pubblicate: le due relectiones metricologiche, l'Epometria (1515) e la Prosodia (1517), quest'ultima accompagnata da 36 epigrammi; il corso accademico (1513-1514) e l'ampio commento (1516) dedicati all'Historia Apostolica, il poema epico di Aratore d'argomento neotestamentario; e il carme teologico Antimoria, edito nel 1536 a Coimbra, con appendice di 50 epigrammi ${ }^{1}$. Del resto, è lo stesso umanista a esplicitare e motivare tale predilezione, in un passo dell'Epometria già citato da Sebastião Pinho:

Soleo, auditores suavissimi, poetas vobis libentius quam historicos vel oratores interpretari: non quod hos inutiles incipientibus arbitrer [...], sed quia, in tanta apud nostros homines linguae latinae incuria, magis necessarium carmen vobis quam prosam orationem multoque esse utilius puto. Sive enim ratio honeste vivendi, sive optime discendi a vobis petatur, utrumque facilius a vatum quam ab oratorum fontibus haurietis ${ }^{2}$.

${ }^{1}$ Per un quadro dell'attività poetica di Barbosa, cf. Sebastião Tavares de Pinho, Humanismo em Portugal. Estudos I. Lisboa, Imprensa Nacional - Casa da Moeda, 2006: 97-114 ("Aires Barbosa, pedagogo e poeta"); sul ruolo assegnato alla poesia dall'umanista portoghese cf. ibid.: 129-147 ("Retórica e poética na Epometria de Aires Barbosa"), in particolare: 132-133. Riguardo a vita e opere dell'autore, cf. José Henrique Rodrigues Manso, Comentário de Aires Barbosa ao segundo livro da Historia Apostolica de Arátor. Lisboa, Fundação Calouste Gulbenkian, 2011: 28-49, e bibliografia ivi citata. Proprio il gentilissimo collega J.H. Rodrigues Manso desidero qui ringraziare, poiché solo grazie alla sua cortese disponibilità ho potuto consultare bibliografia e testi, di pertinenza barbosiana, altrimenti per me inaccessibili.

${ }^{2}$ Arii Barbosae Lusitani Relectio, cui titulus Epometria. Salmanticae, [Juan de Porras] 1515: Aiv (cf. Pinho, Humanismo em Portugal... op. cit.: 132). 
Per il nostro umanista, dunque, lo studio dei testi poetici si fa preferire a quello delle opere in prosa, in quanto miglior veicolo sia all'acquisizione di nuove conoscenze, sia alla conquista di un onesto stile di vita. Più in generale, la scrittura poetica e la sua interpretazione sono così riconosciute come i più efficaci strumenti di elaborazione e trasmissione culturale, sui diversi piani della lingua, della scienza, dell'etica.

Questa gerarchia pedagogico-letteraria barbosiana, per essere compresa nei suoi rapporti con le speculazioni dell'Umanesimo italiano, richiede innanzitutto che si sgombri il campo da un retaggio di pregiudizi negativi duro a morire, circa il ruolo svolto dalla poesia nella cultura italiana, tra Petrarca e Poliziano. Senza risalire alle preclusioni di origine romantica o a quelle estetiche di un Croce, l'incomprensione del fenomeno si trova confermata in un famoso saggio di Carlo Dionisotti, secondo il quale "bisogna rassegnarsi alla constatazione di fatto che senza poesia è il secolo tutto [...], non l'una piuttosto che l'altra lingua"; perfino la generazione di autori come Strozzi, Pontano, Campano, attiva a partire dagli anni '40 (e in realtà tutt'altro che priva di precursori), non avrebbe rappresentato che un'“acerba primavera poetica", che "poté aprirsi a un'età che era stata deserta dalla poesia, in quanto alla stessa data quella stessa età aveva nella prosa affermato per sempre la conquista d'una nuova filologia e d'una nuova storiografia"3. Poiché invece l'età indicata era stata fin troppo feconda di poeti, molti dei quali di notevole seppur misconosciuto valore, un semplice rovesciamento di prospettiva potrebbe legittimare il sospetto che una così persistente svalutazione sia stata favorita, paradossalmente, dallo straordinario successo riscosso dal Rinascimento italiano non tanto presso i critici letterari, quanto presso storici della cultura, del pensiero e delle arti. Da Burckhardt a Baron, da Garin a Kristeller e oltre, la lente degli osservatori si è infatti sempre soffermata, alla ricerca dei fondamenti storici e ideologici del movimento, sulla vigorosa trattatistica dei vari Salutati, Bruni, Biondo, Alberti, Valla, Ficino, Pico; e se opere più propriamente letterarie hanno attirato nuove attenzioni, magari come testimonianze delle voci meno ottimiste di questa cultura, a beneficiarne, non a caso, sono stati ancora generi prosastici, quali l'apologo o il dialogo lucianeo (dalle Intercenales dell'Alberti al Charon del Pontano, antesignani dell'erasmiano Moriae encomium).

Per fortuna, il panorama critico va seppur lentamente cambiando. Il "non secondario contributo" dato dalla poesia alla formazione della cultura umanistica è stato ad esempio rivendicato da Francesco Bausi ${ }^{4}$; ed anche Francisco Rico

${ }^{3}$ Cf. Carlo Dionisotti, Geografia e storia della letteratura italiana. Torino, Einaudi, 1967: 179199 ("Discorso sull'umanesimo italiano") in particolare 185-188.

${ }^{4}$ Cf. "Modi e forme della poesia umanistica (tra latino e volgare)", in G. Rizzo (ed.), 
è giunto a riconoscere che "per gli umanisti la centralità della letteratura non fu solo una teoria del sapere ma, prima ancora, un'esperienza estetica personale [...]. Se Petrarca, Cola di Rienzo, Lovato Lovati, non avessero vibrato di tale passione di artisti, l'Umanesimo non sarebbe mai venuto alla luce"5. Molta strada, tuttavia, resta ancora da compiere. Anche in quest'ultima ricostruzione del "sogno dell'Umanesimo", i documenti di riferimento sono ancora desunti dalla trattatistica sempre citata, mentre alla scrittura poetica non è rivolta alcuna attenzione, se non per qualche rapido accenno al prestigio sociale che il potere poteva derivarne. Il pur ampio spazio riservato a uno straordinario poeta trilingue come Poliziano, maestro del nostro Barbosa, è di fatto interamente dedicato ai Miscellanea, la summa dell'Ambrogini filologo: per dimostrare, oltretutto, che col suo specialistico approccio ai nodi più complessi della critica testuale, Poliziano sancirebbe "l'avvenuto smembramento dell'ideale umanistico del sapere", nonché il ripiegarsi "su se stessa, in una nicchia professorale e professionale", della tradizione storica dell'umanesimo italiano ${ }^{6}$.

Non è questa la sede per argomentare il mio dissenso circa tale interpretazione storica, incurante dei capolavori letterari della generazione del Poliziano (grazie ai quali la passione per le lettere si trasmise a quella successiva, cui appartenne lo stesso Barbosa), e del dibattito sviluppatosi in Italia intorno alla questione del rapporto tra latino e volgare: premessa non al ridimensionamento erudito di metodo e obiettivi del movimento umanistico, ma alla riaffermazione di questi ultimi attraverso i nuovi strumenti espressivi delle lingue nazionali ${ }^{7}$. Per tornare al nostro tema, accertati i limiti e la provvisorietà delle attuali conoscenze, converrà piuttosto riavviare la ricerca dal

L'identità nazionale nella cultura letteraria italiana, Atti del III Congresso nazionale dell'ADI, Associazione degli italianisti italiani (Lecce-Otranto, 20-22 settembre 1999). Galatina, Congedo, tomo 1, 2001: 89-96 in particolare 89.

${ }^{5}$ Cf. Francisco Rico, Il sogno dell'Umanesimo (ed. or. Madrid 1993). Torino, Einaudi, 1998 : 17-18.

${ }^{6}$ Ibid.: 71-74. Non a caso, sempre Rico ("Nebrija, Aires Barbosa et l'Humanisme de leur temps", in L'bumanisme portugais et l'Europe, Actes du XXI Colloque International d'Etudes Humanistes. Paris, Fondation Caluoste Gulbenkian - Centre culturel portugais, 1984: 245-246) dello stesso Barbosa non prende in considerazione se non l'attività di filologo.

${ }^{7}$ Quando infatti si riconobbe che la "grammatica" non era peculiarità del latino, ma struttura profonda della lingua riscontrabile anche nell'idioma materno, fatalmente in Italia quest'ultimo affiancò il latino come lingua di cultura: riconosciuta come "classica", in quanto regolata da norme fondate sull'applicazione dell'umanistico principio d'imitazione. Del resto, a tale approdo contribuì fortemente il fitto e continuo dialogo intertestuale che gli scrittori quattro-cinquecenteschi intrecciarono non solo con l'antichità, o con intramontabili autorità medievali, ma anche con un vasto coro di voci coeve, appartenenti a entrambi gli orizzonti linguistici: dialogo in minima parte finora indagato rispetto alle dimensioni del fenomeno, e al quale ho rivolto la mia attenzione in vari studi, tra cui mi permetto qui di citare «La fonte d'ogni eloquenzia». Il canzoniere petrarchesco nella cultura poetica del Quattrocento ferrarese. Roma, Bulzoni, 2002. 
ruolo che alla poesia assegnarono i diretti interessati; in questa sede partendo, naturalmente, dalle posizioni assunte in merito dallo stesso Barbosa, come quelle inserite nella Praefatio al suo carme Antimoria:

Quae dignior, quae uberior actio homini facundo dari potest [...] tractanda, quam ea quae pro Sapientia pugnans Iesum laudet, et Insaniam oppugnans a vitiis absterreat? [...]. Quamvis enim Moriae encomium summa cum delectatione ab eruditis legatur, intelligentibus scilicet leporem illum suavissimum ipsis innoxium, tamen ab aliis, quae turba maxima est, non sine noxa legi potest [...]. Erasmus undanti solutae vocis eloquio qua libitum est illi digredi excurrit; nobis placuit prudentium imitari carmen scribentibus, qui pedum numeris astricti libere divagari non possumus [...]. Tamen expetivimus Christo Optimo Maximo officii genere aliquo placere et in hoc secessu tenui avena sylvestrem musam, id est inconditos versiculos meditari ${ }^{8}$.

Consapevole della propria inferiore eloquenza, non per questo Barbosa si presenta meno combattivo nel voler riaffermare, con mezzi letterari, i valori assoluti della Sapienza cristiana: virtù divina, antitetica a quella Follia così ambiguamente lodata da Erasmo. Si trattava, in realtà, di una missione costante della sua attività letteraria, già indicata nella Praefatio al suo Commento del libro I dell'Historia apostolica di Aratore:

Denique ut enarres eum poetam obsecramus, cuius sacrum carmen non tandem ad ingenni cultum, verum etiam ad pietatem veramque sapientiam pertineat [...]. Cunctos ad pietatem instruit et theoseuiam (sic), quae hominis est (ut tolerantissimus heros Iob retulit) Sapientia?.

Nella Praefatio al carme Antimoria, ben attento alle implicazioni sottese alla scelta del genere, Barbosa sostiene inoltre l'opportunità di affidarsi non alla sregolata "favola in prosa" (lucianea) del suo antagonista, ma all'equilibrio proprio della poesia: nemica delle divagazioni grazie ai freni del metro, e qui connotata come silvestre, umile, e dunque più adatta a quella maxima turba cui

${ }^{8}$ Il testo, dopo la princeps Arii Barbosae Lusitani Antimoria. Eiusdem nonnulla Epigrammata. Conimbriae, apud Coenobium divae Crucis, 1536, è stato pubblicato e tradotto da José Pereira Tavares, "Tradução do poema Antimoria e dalguns epigramas de Aires Barbosa", in Arquivo do distrito de Aveiro, 26 (1960) 9-82, da cui si cita (: 26, 28); riscontrandolo comunque con l'ed. antica (: IVv, vv-VIr), e tenendo conto della revisione di José V. de Pina Martins, Humanismo e erasmismo na cultura portuguesa do século XVI. Estudos e textos. Paris, Fundação Calouste Gulbenkian - Centro Cultural Português, 1973: 197-220.

${ }^{9}$ Aratoris cardinalis Historia apostolica cum commentariis Arii Barbosae Lusitani. Salmanticae, in aedibus Ioannis de Porris, 1516: c. Aiir. Soffermando la propria attenzione sul concetto di pietas, e rilevando le affinità con la poesia bucolica di Battista Spagnoli (il Mantovano), citò già il passo Jorge Alves Osório, O humanismo português e Erasmo, Dissertação de doutoramento. Porto, Faculdade de Letras da Universidade do Porto, 1978, tomo I: 54. 
l'equivoca ironia erasmiana risulterebbe assai pericolosa. In realtà poi il carme, incentrato sul grandioso contrasto tra l'azione perniciosa di Moria (scesa tra gli uomini a guidare l'esercito dei vizi) e quella civilizzatrice e salvifica della Sapienza divina, potrebbe effettivamente apparire lucretianae phraseos foeliciter aemulum, com'è definito nell'elogiativa prefazione da Jorge Coelho ${ }^{10}$. Più propriamente tuttavia, data la brevità dei contenuti e la programmatica sobrietà della forma (minimo ad esempio è il ricorso a riferimenti mitologici), il carme di Barbosa sembra piuttosto assumere i caratteri di una selva, il genere poetico recentemente rilanciato, non a caso, dalle quattro grandi prove del Poliziano ${ }^{11}$.

Proprio come in quest'ultime, di tale duttile genere l'Antimoria offre infatti un'interpretazione etico-didattica, che è particolarmente interessante riscontrare in alcuni versi:

\section{Non bic lascivos iuvenum cantamus amores aut Graiis nocuit quantum implacatus Achilles, Moria sed quantum noceat mortalibus una (vv. 88-90). \\ Moria mortales animos sic turbat, ut id quod est verum estque bonum, quamvis videantque probentque, deteriora sequi impellat [...] (vv. 177-179). \\ O Corydon, Corydon, coeli mysteria nescis, et Domini ignoras super omnia tempora vitam (vv. 370-371)12.}

Troviamo qui esplicitati alcuni punti strategici della poetica di Aires Barbosa. In primo luogo, si evidenzia il rifiuto della lasciva tematica amorosa (espresso con abile rovesciamento di un verso di Marziale) ${ }^{13}$, che ben si sposa

${ }^{10}$ Cf. Tavares, "Tradução do poema Antimoria...” op. cit.: 22 (Arii Barbosae Lusitani Antimoria..." op. cit: Iv). Altro letterato, Jorge Coelho, molto sensibile ai temi della fede, se di lì a quattro anni pubblicherà a sua volta un poemetto esametrico De patientia christiana, accompagnato da una Lamentatio divae Mariae Magdalenae (cf. Jorge Coelho, De patientia christiana liber unus. Item nonnulla alia quae in fine videbis. Lisboa, apud Ludovicum Rothorigum, 1540: cc. $3 r-16 r$ e $16 v-18 r$ ).

${ }^{11}$ Uscite a stampa tra il 1482 e il 1491, e oggi disponibili nell'ottima edizione (Angelo Poliziano, Silvae, a cura di F. Bausi. Firenze, Olschki, 1996). Riguardo ai rapporti intrattenuti dal Poliziano con i suoi numerosi allievi portoghesi, e con lo stesso re D. João II, rimando a Vincenzo Fera, Una ignota expositio Suetoni del Poliziano. Messina, Centro di studi umanistici, 1983: 17-22 (segnalatomi dalla prof.ssa Rita Marnoto, che ringrazio) e a Rita Biscetti, "La gloria dei Portoghesi: ancora sull'epistola di Poliziano a D. João II”, in Humanitas, 43-44 (19911992): 291-303.

${ }^{12}$ Vd. Tavares, “Tradução do poema Antimoria...”, op. cit.: 38, 42, 50 (Arii Barbosae Lusitani Antimoria... op. cit.: IXv, XIIr, XVIIr).

${ }^{13}$ Cf. Mart. Epigr. 14. 187. 1: Hac primum iuvenum lascivos lusit amores. Anche la clausola del v. 88 richiama formule classiche, soprattutto ovidiane (Rem. 379: cantet amores; Epist. 15. 155: cantat amores; Epist. 16. 257: cantabam ... amores), poi riprese soprattutto da Nemesiano (cf. Ecl. 4. 3: cantabat amores); ma un precedente identico c'è solo in Boccaccio (Egl.13.131) e in Gabriele Altilio (Carm. 1. 59). Per le edizioni di riferimento tanto degli autori classici quanto di quelli 
con la successiva identificazione del morus (vv. 178-179) nel tipo umano consapevole del bene, ma incapace di opporsi alla tentazione (prevalentemente, per l'appunto, di natura erotica): tipo umano il cui archetipo era stata la Medea ovidiana (Met. 7.20-21: video meliora proboque / deteriora sequor), ma che si era ormai ben più esemplarmente riproposto nei versi di Francesco Petrarca (Rvf 264, 136: “et veggio 'l meglio, et al peggior m'appiglio"), per poi riverberarsi tra i già numerosi emuli di quest'ultimo, nell'affollato panorama della poesia italiana, non solo volgare: notevoli in particolare le riprese di due poeti latini che vedremo importanti nella formazione di Aires Barbosa, il ferrarese Tito Vespasiano Strozzi (Eroticon 1. 8. 56: Optima cum videam, deteriora sequar) e il fiorentino Ugolino Verino (Flametta 2. 22. 43: Et meliora vident et deteriora sequuntur $)^{14}$. In secondo luogo, l'accostamento citazionale del morus al virgiliano pastore Coridone (Ecl. 2. 69: A, Corydon, Corydon, quae te dementia cepit'), un personaggio ancora una volta "folle d'amore", vale a confermare, seppur con accenno isolato, la connotazione silvestre (e dunque umile) di questo canto, come preannunciava, con altrettanto vistosa ripresa virgiliana (Ecl. 1. 2: silvestrem tenui musam meditaris avena), il già ricordato passaggio prefatorio (expetivimus [...] tenui avena sylvestrem musam, id est inconditos versiculos meditari).

Se Aires Barbosa rivela di saper abilmente ricorrere, per dichiarare la propria poetica, a locuzioni elaborate dalla latinità classica, non meno attento egli si mostra nei confronti dei più importanti poeti dell'umanesimo italiano. Pur all'interno di un verso (89) quasi interamente estrapolato da Orazio (Epist. 2.2.42: Iratus Grais quantum nocuisset Achilles), risalta ad esempio l'inserimento di implacatus, un aggettivo raro nel latino classico (una occorrenza al femminile in Virgilio e Ovidio), e declinato al maschile, anche tra i poeti umanisti, dal solo Poliziano, e proprio in quei Nutricia (Silve 4. 116) che vedremo capaci di incidere un segno profondo nella poetica barbosiana. Inoltre, se la clausola del v. 90 (mortalibus una) è prettamente lucreziana (cf. Rer. nat. 2. 919), essa si fonde nel secondo emistichio con una formula (noceat mortalibus) che non ha altri precedenti se non in Battista Spagnoli (Sylvae 2. 5. 3: Sive quod aetherei noceant mortalibus ignes), altro poeta italiano di cui è già stato notato il forte

rinascimentali, rimando all'archivio digitale di cui mi sono servito per il reperimento dei riscontri: Musisque deoque. Un archivio digitale di poesia latina (www.mqdq.it/mqdq/index.jsp); e Poeti d'Italia in lingua latina, tra Medioevo e Rinascimento (www.mqdq.it/mqdq/poetiditalia/index.jsp).

${ }^{14}$ Entrambi gli autori inserirono l'espressione nei loro canzonieri amorosi; lo Strozzi, in particolare, l'adottò in un'elegia risalente agli anni 1447-50 (cf. Anita Della Guardia, Tito Vespasiano Strozzi. Poesie latine tratte dall'Aldina e confrontate coi Codici. Modena, Blondi \& Parmeggiani, 1916: 20), su cui tornerò. I Rerum vulgarium fragmenta (= Rvf) si citano dall'edizione di Petrarca, Canzoniere. Rerum vulgarium fragmenta, a cura di R. Bettarini. Torino, Einaudi, 2005. 
influsso sull'ispirazione religiosa di tanti carmi di Barbosa ${ }^{15}$. Al v. 175 , il sintagma Mortales animos può ricordarne di simili in Orazio (Sat. 2. 6. 94: mortales animas) e Silio Italico (Pun. 2. 316: mortale animi), ma più da vicino (anche per il contesto) esso richiama il v. 253 di una famosa e già citata elegia di Tito Strozzi (Eroticon 1. 8: Mortales animos in scelus omne trabit), su cui non a caso dovremo tornare. Priva di precedenti classici è, al v. 370, l'espressione coeli mysteria, che a termini solo invertiti figurava nel solito Tito Strozzi (Borsias 4. 132: mysteria coeli) e in Ugolino Verino (Carlias 8. 862), il quale peraltro l'aveva utilizzata anche con identica successione verbale, ma significato capovolto (Carlias, App. 1.298 e 1.958: celi et mysteria nosces) ${ }^{16}$.

Tali aspetti del carme Antimoria si ripresentano perfettamente nella silloge di epigrammi che accompagna il poemetto (e più in generale nella complessiva produzione in versi dell'autore $)^{17}$. L'amore vi è escluso, se si eccettua la lode di quello coniugale nella triste occasione dell'Epitaphium uxoris: per comporre il quale di nuovo Barbosa si rivela padrone di un'ampia cultura poetica, come mostrano memorie tanto antiche (Lucano, Silio Italico, Stazio, Giovenale, Ausonio, Prudenzio: con tutta polizianea attenzione per l'età argentea e tardoantica) quanto moderne (Pontano, Verino) ${ }^{18}$. Varie deplorazioni, viceversa, sono rivolte a Issa, una fanciulla dai costumi sfrontati. I testi più ambiziosi sono d'argomento politico: alcuni seguono la rivolta delle Comunidades di Castiglia (1520-1521), con sguardo dapprima favorevole (finché essa pare avere come obiettivo la politica di Carlo V) poi contrario (quando assume caratteri antiaristocratici); altri inneggiano alle

${ }^{15} \mathrm{Cf}$. Osorio, O bumanismo português... op. cit.: 54-56.

${ }^{16}$ Tempora vitam, a sua volta, è clausola assente nel latino antico, mentre vanta occorrenze nei duecenteschi J. Stefaneschi (due) e A. Mussato, nonché nei quattrocenteschi M. Andronico e F. Andrelino. Tuttavia sulla formula barbosiana può aver influito l'affine tempora vitae, al contrario molto frequente nella classicità (attestazioni in Virgilio, Ovidio, Manilio, Seneca, Lucano, Stazio, ecc.) come nella poesia umanistica (Petrarca, Basinio da Parma, Tito Strozzi, Braccesi, Pontano, Verino, Spagnoli, Poliziano, ecc.).

${ }^{17}$ Per una puntuale illustrazione di queste tematiche, cf. Pinho, O humanismo português... op. cit.: 109-112. Oltre al carme Antimoria, diversi degli epigrammi qui ricordati sono stati èditi modernamente e tradotti da Tavares, "Tradução do poema Antimoria... " op. cit.: 68-77.

${ }^{18}$ Questo il testo: Hic iacet Elisabet generosae stirpis, et uxor / Barvosae. Moriens morte beata fuit: / nam bene quae vixit, fatali molliter hora / castam animam coelo reddidit, ossa solo. / Est igitur foelix: nullum facunda Solonis / foelicem ante obitum dicere vox solita est. / Rapta licet fuerit nimium florentibus annis, / te patrem quino pignore fecit, Ari (Tavares, "Tradução do poema Antimoria..." op. cit.: 68-69; Arii Barbosae Lusitani Antimoria... op. cit.: xxvinr). I modelli, nel dettaglio, 1 generosae stirpis: Prud. Symm. 1. 176; 2 beata fuit: Pontano De am. coniug. 2. 7. 30; 3 fatali hora: Lucan. Phars. 9. 87, Stat. Theb. 8. 185, Verino Carlias 8. 506; 4 animam coelo reddidit: Stat. Theb. 8.324 (efflare animas et reddere caelo); ossa solo: Prop. El. 1. 22. 8, Sil. Ital., Pun. 6. 160; Pontano De tumulis, 1. 5.7 e 1. 17. 3; 5-6 facunda Solonis ... vox: Iuven. Sat. 10. 274; 6 foelicem obitum: Verino Epigr. var. 7 (In obitu Phylippae Corsinae), 31; 7 Rapta ... florentibus annis: Auson. Parent. 7.5 (Raptus ... florentibus annis); Verino Epigr. var. 6 (Epit. Ursini Lanfredini), 3-4 (florentibus annis / raptum). 
battaglie della Spagna, pacificata contro i Francesi; altri ancora condannano l'inerzia delle potenze cristiane contro l'avanzata dei Turchi. Le esperienze personali evocate riguardano in primo luogo l'insegnamento: esortazioni agli studenti, difesa delle discipline linguistiche; i versi satirici colpiscono sempre il mondo degli studi, con frecciate contro i grammatici litigiosi, gli astrusi legisti, gli astrologi.

Quando subentrano meditazioni più intime, emergono aspettative andate deluse, e il tono talvolta si fa malinconico; proprio tra questi carmi s'incontra il più interessante nella nostra prospettiva, la $A d$ se paraènesis: dove di nuovo viene ripresa una caratteristica metafora petrarchesca, raffigurante l'io del poeta come barca in balìa delle onde, per essere però completamente ribaltata, a riaffermare l'efficacia della ragione e della virtù nel contrastare le avversità della vita ${ }^{19}$ :

Rector ut in tumido puppim regit aequore, gnarus
quos vitet scopulos, quae loca tuta petat,
frangit et hanc, huic cedit aquae, moderamine clavi
infirmam tabulam per freta longa vehit;
sic et, Ari, motus animi fluctusque tumentes
nunc preme, nunc laxa, sub ratione tenens,
indomitumque salum vitae virtute gubernans:
sic supera curas, ut maris ille minas ${ }^{20}$.

Anche in questo caso, peraltro, la cultura poetica di Aires Barbosa si conferma ben sostenuta da letture tanto classiche quanto moderne. Il sintagma tumido aequore, ad esempio, se aveva alle spalle il tumida aequora di Virgilio (Aen. 1. 142), figurava identico nell'Urania di Giovanni Pontano (5. 328). Puppim regit si leggeva in Rutilio Namaziano (De red. 1.456). Vitet scopulos (al v. 2) non ha precedenti classici, mentre ricorda da vicino (pur senza il petrarchesco valore metaforico) espressioni come vitare [...] / tempestates scopulosque latentes di Tito Strozzi (Borsias 3. 154-155), o vitare obstantis scopulos di G.A. Augurelli (Serm. lib. 1. 9.3). Di certo, la clausola loca tuta petat, se può denunciare memoria di Corippo (Iohannis 2. 223: loca tuta petens), o più da lontano di Ovidio (Trist. 4. 1. 19: loca iussa petentem), ha alle sue spalle una reiterata formula strozziana: loca sola peto (Erot. 2. 5. 10), loca sancta petat (ivi 3.1.42), loca sola petunt (ivi 4. 23. 20), a sua volta trasposizione latina di $R v f 35,1-2$ ("Solo et pensoso i più

${ }^{19} \mathrm{Cf}$. ad es. Rvf 189: "Passa la nave mia colma d'oblio / per aspro mare, a mezza notte il verno [...]; / la vela rompe un vento umido eterno / di sospir', di speranze e di disio [...]; / morta fra l'onde è la ragione e l'arte, / tal ch'incomincio a desperar del porto".

${ }^{20}$ Tavares "Tradução do poema Antimoria... " op. cit.: 76-77 (Arii Barbosae Lusitani Antimoria... op. cit.: $\mathrm{xxxx} r$ ). 
deserti campi / vo mesurando"). Staziana è la clausola del v. 3, in realtà in gran parte ispirato da Theb. 10. 183 (Fregit iter, subit ad vidui moderamina clavi); mentre marcatamente ovidiana è l'espressione per freta longa (v. 4), cinque volte utilizzata dal poeta di Sulmona (Her. 7. 46; Met. 7.67 e 8.142; Fasti 3.868 e 5. 660): numerose di conseguenza le riprese umanistiche. Con ordinamento inverso dei due termini, motus animi (v. 5) è locuzione già lucreziana (Rer. nat. 4. 1072) e oraziana (Ars poet. 111); ma nella già incontrata Urania del Pontano, essa figura proprio nella successione adottata da Barbosa (3.558), oltre che in quella anticamente attestata (2.828), peraltro adottata anche da Battista Spagnoli (Sylvae 1. 10. 19). La clausola fluctusque tumentes è probabilmente memore di Virgilio (Aen. 7. 818: fuctu suspensa tumenti), e più da vicino di Sedulio (Carm. pasch. 3. 223: fluctuque tumenti); ma un precedente identico ha in Boccaccio (Egl. 11. 186), e uno molto simile in Tito Strozzi (Erot. 1. 6, 65: fuctus placare tumentes). Nunc preme era formula già di Valerio Flacco (Argon. 1. 334), che ne adattava altre precedenti, leggermente diverse, di Tibullo, Seneca, Lucano, Stazio; e proprio da Stazio (Theb. 3. 392-393: animosaque pectora laxet / sera quies: nobis dolor haud rationis egebit) sembra essere stato suggerito quanto resta del v. 6. Peraltro la clausola del verso, pur senza perfetta coincidenza (ratione tenere/ teneri/tenentur), è tipicamente lucreziana, con ben quattro occorrenze (Rer. nat. 1. 948, 1. 1081, 3. 572, 4.23) già rielaborate, nella forma ratione tenenda, da Basinio da Parma (Carm. var. 20. 38) e Giovanni Pontano (Meteororum liber, 853). La clausola del v. 7, virtute gubernans, nella letteratura antica ha il solo precedente di Optaziano Porfirio (Carmina 15.2), sicché possono forse essere più significativi i precedenti moderni di Matteo Zuppardo, Alfonseis, X 84 (virtute gubernat) e del fiorentino Naldo Naldi, Eleg. ad Laur. Medicen, 3.1 .39 (virtute gubernes). Infine, il paragone conclusivo aveva un precedente in Virgilio, con Mezenzio a sostituire il marinaio (Aen. 10.693696: Ille velut rupes [...] / vim cunctam atque minas perfert caelique marisque); ma non trascureremo che, riferito all'amata, lo aveva anche in Tito Strozzi (Erot. 5. 2. 21-22: Ipsa immota tamen perstat, velut aspera cautes / quae maris assiduas negligit alta minas).

Se le componenti classiche e umanistiche della scrittura poetica barbosiana potevano rappresentare un dato prevedibile (ma ugualmente da sottoporre a verifica), non meno intuibile e coerente, rispetto al ruolo eminente che l'Umanesimo italiano assegnò al genere poetico, è da considerarsi il rilievo attribuitogli da Aires Barbosa (come visto in apertura) quale strumento di conoscenza e formazione. Più in particolare, pur comprendendo testi composti attraverso vari decenni, la produzione letteraria barbosiana mostra rilevanti caratteri di continuità con le tendenze letterarie prevalenti a Firenze negli anni in cui il nostro umanista vi frequentò la scuola del Poliziano (14901495). Prima però di approfondire tali aspetti, appare opportuno rilevare come 
essa nascesse da precise scelte di campo tra le diverse posizioni estetiche che avevano alimentato il dibattito umanistico sulla poesia: concezioni che proprio nella riflessione dell'Ambrogini avrebbero trovato la loro massima sintesi, e che necessariamente richiedono ora un veloce riepilogo.

Non mi soffermerò sulle celebri, inaugurali discussioni affrontate sul tema da Petrarca, che nelle Invective contra medicum aveva proclamato primos nempe theologos apud gentes fuisse poetas; da Boccaccio, che tale principio aveva riaffermato nelle Genealogie deorum gentilium; e da Coluccio Salutati, che nel De laboribus Herculis aveva riconosciuto alla poesia il ruolo di arte civilizzatrice, in quanto discorso figurato atto a rappresentare sotto veste allegorica verità ineffabili, soprannaturali (poetica theologia). Basterà qui rimarcare come tale approccio necessariamente promuovesse una poesia dai contenuti elevati (morali o politici), da affidare a generi altrettanto elevati (epica, tragedia), o almeno disponibili a sovrasensi allegorici (bucolica) o alla comunicazione colta (epistole metriche). Ebbene, tra le più lucide testimonianze di questo orientamento estetico, particolarmente illuminante considero quella proveniente da un centro culturale cui la stessa Firenze guardò sempre con molta attenzione (ne fu originario Savonarola, tra gli altri), e anch'esso fortemente attrattivo nei confronti di dotti portoghesi. Mi riferisco naturalmente a Ferrara, dove come giurista presso lo Studio operò Ludovico Teixeira, e dove tra il 1497 e il '99 soggiornò Henrique Caiado, già allievo del Poliziano a Firenze, di Filippo Beroaldo a Bologna, ma ora in contatto con numerosi letterati ferraresi, a cui indirizzò molti epigrammi: e tra i destinatari non mancò quel Tito Vespasiano Strozzi che, oltre a comporre opere poetiche che già sappiamo capaci di lasciar tracce importanti nella scrittura poetica di Aires Barbosa, assieme al figlio Ercole dedicò vari epigrammi allo stesso Teixeira ${ }^{21}$. Molto importanti anche per i poeti portoghesi formatisi a Firenze e Ferrara (come Caiado e il nostro Barbosa, poco o nulla propensi al canto amoroso, e dediti invece a quello di soggetto morale, politico, religioso), risultarono dunque certamente le dispute manifestatesi alla celebre scuola ferrarese di Guarino da Verona.

Le teorie letterarie del maestro sono ben testimoniate, oltre che dal suo epistolario e dagli scritti didattici, da un documento fondamentale quale è la Politia litteraria di Angelo Decembrio, uno dei tanti umanisti formatisi alla sua scuola. Raffinato dialogo, in cui tutti i componenti del circolo guidato da

${ }^{21}$ Per i rapporti tra gli Strozzi e il Teixeira rimando a Vincenzo Fera, Una ignota expositio... op. cit.: 20. Quanto alle frequentazioni ferraresi di Henrique Cayado, nell'ed. Henrique Caiado, Aeglogae et sylvae et epigrammata Hermici. Bononiae. Benedictus Hectoreus [Faelli], 1501, si incontrano epigrammi dedicati a Tito Strozzi (Kır $r$, Kvır $r$ ), Ercole Strozzi (Hvr-v, Kıv), Celio Calcagnini (Kviv), Luca Ripa (cc. Giıv, Hır, Hııv, Hıvr, Hvv, Mııv), Alberto Tassino (c. Giıv), Gabriele Tassino (Hıır, Hvr), e altri. Tra i non ferraresi, non manca il Poliziano (c. Gviv, Hiıv). 
Guarino e da Leonello d'Este dialogano sulle più varie questioni culturali, l'opera (intrapresa negli anni' 40 e conclusa intorno al 1463) affida le valutazioni di merito soprattutto al marchese-umanista, che sintetizza il pensiero estetico guariniano nella seguente regola aurea:

Decet enim in primis ea librorum volumina perquirere, quae hominem efficiant eloquentem et industrium, a naturali usu minime discedentem ${ }^{22}$.

Tale principio non comportava una svalutazione della poesia. Il rischio di desumerne criteri di giudizio angustamente utilitaristici era certo presente, e infatti nell'opera non manca chi ne deduce la superiorità di Tito Livio rispetto a Virgilio. Leonello risponde tuttavia non solo rivendicando la grande ricchezza informativa delle opere virgiliane, ma anche illustrando i superiori valori che pongono la poesia su un piano privilegiato rispetto alle altre scritture letterarie, sia quanto ad altezza d'ispirazione che per efficacia didattica:

Nec enim a poetis primum incepisse pigebit. Quorum eo altiora vigent ingenia, quo magis animum delectare videntur musico quodam artificio, et, si dicifas est, harmoniae caelesti consimilia. Dicimus autem egregios non omnis generis poetas. Unde merito eruditissima carmina scribentibus poetae nomen inditum est, quod 'factorem' vel 'auctorem' et fere 'primarium' indicat, et ipsum poema 'facturam' vel 'opus'. Ac ipsum

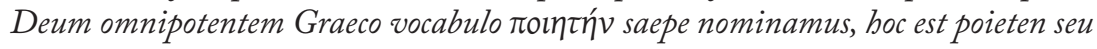
poetam, quamvis pro more sonoque Latine 'factorem' magis interpretamur. Huicque divino pioque hominum generi, non irreligioso, ut stultum vulgus existimat, laurea dicata aliaque ab imperatoribus munia donari solita. Ad hoc in lectione carminis iis, qui metricam et solutam tractant orationem, voluptas accedit maior, faciliusque carmen memoria retinetur et imbibitur, quo sensim animus praecipue iuvenilis cum delectatione simul in sermonum gravitate nutritur ${ }^{23}$.

Il circolo guariniano, dunque, attribuiva ai poeti non solo il dono di esprimersi in forme didatticamente utili perché più facilmente memorizzabili, ma soprattutto un ingegno in possesso di una creatività a suo modo divina, in virtù della musicalità, simile all'armonia dei cieli, che ne pervade le opere: immagini che ritroveremo nello stesso Poliziano. Tuttavia, proprio l'eccezionale natura e l'alta funzione educativa attribuite alla poesia non rendevano possibile, nella Politia litteraria, un apprezzamento che prescindesse da contenuti formativi;

${ }^{22}$ Angelo Camillo Decembrio, De politia litteraria, ed. N. Witten. München-Leipzig, K.G. Saur, 2002: 152 (1. 3. 12). L'intera pars tertia (: 148-159) è dedicata al tema che qui ci interessa: Qui modus ordoque servandus in curanda poliendaque bibliotheca, deinde quo pacto struenda, scilicet qui libri in ea ex Latinis et Graecis opportuni, ac primo de metricis auctoribus, inter quos de Virgilio praecipua mentio, ac Tullio officiorum atque Salustio per Leonellum.

${ }^{23}$ Ibid.: 153 (1. 3. 14). 
in particolare, quell'aderenza alle esperienze concrete della vita (di una vita orientata da forti valori umani e civili), che rendeva imprescindibile la lettura di Virgilio, Terenzio e Giovenale, non trovava riscontro nella frivola poesia lirica ed elegiaca. I maggiori esponenti di questa sono perciò frettolosamente ricordati solo perché si ritiene doveroso prestare attenzione a quegli autori che gli antichi ritennero degni di lode ${ }^{24}$.

Una prima alternativa a questo sistema estetico, per diversi aspetti ben attivo (come visto) nella stessa poetica barbosiana, si presentò nel 1429, grazie all'azione congiunta di un giovane poeta siciliano, Giovanni Marrasio, che emigrato a Siena vi compose una raccolta elegiaca dedicata ad Angela Piccolomini (l'Angelinetum), e dell'umanista che in quegli anni stava traducendo in latino molti dialoghi di Platone (in particolare il Fedro), ossia Leonardo Bruni: ai quali si dovette una nuova teorizzazione della natura divina dell'ispirazione poetica (appunto su base platonica), con esplicita rivendicazione della superiorità di una poesia generata da sacro furore (anche se limitata ad apparentemente futili temi amorosi) rispetto ad un'altra magari dai contenuti più alti, ma dettati dalla ragione ${ }^{25}$. Riprendendo infatti un accenno compreso nell'elegia introduttiva dell'Angelinetum, a lui indirizzata (Indulgere velis nostro, Arretine, furori, / sive sit ille furor, sive sit ille dolor, vv. 2122), il Bruni ne approfondì le implicazioni filosofiche, esplicitando il principio estetico sopra indicato:

non enim omne opus poema est, ne si versibus quidem constet, sed illud praestans, illud hac honorata nuncupatione dignum quod afflatu quodam divino emittitur. Itaque quanto vaticinium coniectationi dignitati praestat, tanto poema, quod ex furore fit, sanorum hominum artificio est anteponendum.

Non solo. Sempre su basi platoniche (ma non senza apporti già stilnovistici), il Bruni aggiungeva come tale furor fosse precisamente da identificarsi con l'ispirazione d'amore:

Haec igitur vehemens occupatio animi atque correptio amor vocatur [...]. Sed unum scias volo, me non tam tibi eximiam hanc palmam esse tribuendam existimare quam

${ }^{24}$ Cf. ibid.: 157-159 (1.3.25-28).

${ }^{25}$ Segnala Donatella Coppini (“L'ispirazione per contagio: 'furor' e 'remota lectio' nella poesia latina del Poliziano”, in Vincenzo Fera - Mario Martelli (edd.), Agnolo Poliziano, poeta scrittore filologo. Firenze, Le Lettere, 1998: 127-164) come sia significativo "che il concetto di furor, in Platone comunque funzionale alla svalutazione della poesia, almeno su un piano gnoseologicoepistemico, sia assunto nell'Umanesimo a fondamento della proclamazione dell'eccellenza della stessa poesia [...]: troppo funzionale si presentava la categoria di furor, troppo appropriata l'immagine di una poesia di natura e origine divina, perché tali nozioni non venissero davvero entusiasticamente assunte" (: 131). Sul tema, cf. anche Raoul Bruni, Il divino entusiasmo dei poeti. Torino, Aragno, 2010. 
Amori. Ille est enim qui verba tibi dictat, qui sententias ostendit, qui varietatem et copiam et elegantiam subministrat ${ }^{26}$.

In realtà, questa promozione di Amore al ruolo di divinità ispiratrice della poesia più compiuta, teorizzata dal Bruni con almeno un ventennio d'anticipo rispetto al contributo decisivo di Marsilio Ficino, sarebbe stata accolta con alterno favore. Di certo, tuttavia, essa rappresentò un presupposto fondamentale del fiorire dell'elegia neolatina sin dagli anni '40, ad opera di giovani autori che non aspettavano altro per sganciarsi dalle convinzioni estetiche dei loro maestri, orientate a sottomettere rigidamente la poesia a finalità etiche e politiche. Si trattò, beninteso, di un affrancamento provvisorio, perché quegli stessi giovani (come Tito Vespasiano Strozzi o Basinio da Parma) non si sarebbero sottratti, più avanti, a cimentarsi con l'epica, il genere poetico più rispondente all'esigenza, mai tramontata, di una poesia civilmente impegnata. Sta di fatto che, per tornare al nostro esemplare contesto ferrarese, nel quale non a caso proprio il Marrasio si trasferì nel 1432, i più talentuosi allievi di Guarino decisero di esordire poeticamente all'insegna dell'elegia, talvolta con espliciti omaggi al poeta moderno che l'aveva sdoganata: è il caso ad esempio di Basinio da Parma, che negli anni del suo studentato ferrarese (1446-49) compose una raccoltina elegiaca (Cyris), introducendola con una inequivocabile citazione del tema del furor ${ }^{27}$.

Partendo da tali premesse, ben maggiore rilievo avrebbe tuttavia assegnato Basinio al ruolo del poeta (seppur sempre su base platonica) nel capolavoro composto dopo il trasferimento alla corte riminese di Sigismondo Pandolfo Malatesta (1449): il Liber Isottaeus, uno splendido romanzo epistolare elegiaco, molto fortunato ai suoi tempi, in cui quattro personaggi (Sigismondo, Isotta, il Padre della fanciulla e il Poeta) si indirizzano trenta epistole metriche equamente suddivise in tre libri, in apparenza celebrative dell'amore tra i due protagonisti, in realtà orientate a una profonda riflessione sul destino umano (tra amore, morte e prospettive ultraterrene), sviluppata in una complessa dialettica tra l'ottimismo filosofico del Poeta e le dolenti perplessità

${ }^{26}$ Traggo questo e i precedenti brani da Giovanni Marrasio, Angelinetum et carmina varia, ed. G. Resta. Palermo, Centro di studi filologici e linguistici italiani, 1976: 144-48. Sempre dal Bruni, nella prefazione al proprio commento a Virgilio del 1462, Cristoforo Landino avrebbe mutuato le precise parole per indicare l'assimilazione di vaticinio e poesia (cf. Coppini, "Lispirazione per contagio..." op. cit.: 138-139).

${ }^{27}$ Nec minor in nobis furor est, si credis amanti, / quam fuit in vestro pectore caecus Amor [...]. I Et pereo, fateor, neque enim celare furorem / posse reor: medio stat mea flamma foro (Cyris, I, 27-28, 35-36; cf. Le poesie liriche di Basinio: Isottaeus, Cyris, Carmina varia, a cura di F. Ferri. Torino, Chiantore, 1925: 78). Per un profilo biografico del poeta, cf. Augusto Campana, "Basinio da Parma", in Dizionario biografico degli italiani. Roma, Istituto della Enciclopedia Italiana, vol. 7, 1965: 89-98. 
di Sigismondo e Isotta ${ }^{28}$. Lo scenario dell'opera, in effetti, è estremamente malinconico. Sigismondo soffre i continui distacchi dall'amata (I 1) dovuti ai suoi impegni di capitano di ventura, e, dopo numerosi travagli, ne subisce impotente la morte (III 7); non trovando alcuna consolazione nel mirabile sepolcro che (come nella vita reale) le aveva predisposto, si propone di ricorrere ad arti magiche, pur d'incontrare lo spettro della fanciulla (III 9). Ancora più infelice Isotta: spesso sola (I 2), non ha una fede che la conforti, e cerca una speranza d'eterno nelle parole del Poeta (II 1); avverte che perderà il bambino che ha in grembo, vittima innocente delle proprie colpe (II 5), e in effetti così accadrà (nel racconto come nella vita), pochi giorni dopo il parto (II 6); e ben poco confortata dal sepolcro allestitole (III 6), muore disperata, imprecando contro gli dèi crudeli (III 8: nella finzione poetica, visto che storicamente, invece, ella sopravvisse diversi anni al proprio amante e poi sposo).

Dinanzi a tanto dolore, solo il Poeta cerca di dare, con ogni argomento, prospettive e significati positivi: suo compito nel corpo del racconto è appunto quello di distribuire sapienza, anche se con parziale successo ${ }^{29}$. Così, ad Isotta che gli chiede se la vita si protragga dopo la morte, e con essa l'amore per le persone care, il Poeta risponde affermativamente (2.2), basandosi su una concezione dell'anima come principio di movimento che risaliva al $\mathrm{Fedro}^{30}$, ma negando, sulla base della non inferiore testimonianza di un poeta, Virgilio, quella teoria della metempsicosi (attribuita a Pitagora) che avrebbe comportato come ineludibile corollario l'oblio delle esperienze terrene ${ }^{31}$. E ugualmente a Platone, Omero e Virgilio (tutte voci divinamente ispirate) si affida il Poeta nel rassicurare Sigismondo quando il principe, disperato per la morte di Isotta,

${ }^{28}$ Ho trattato più distesamente questi temi nello studio Italo Pantani, Responsa poetae. Corrispondenze poetiche esemplari dal Vannozzo a Della Casa. Roma: Aracne, 2012: 133-171 ("L'approdo al romanzo epistolare").

${ }^{29}$ Del resto, si ricorderà che Sigismondo fu scomunicato per eresia da papa Pio II (il grande umanista E.S. Piccolomini), con l'accusa di negare l'immortalità dell'anima e la resurrezione dei morti, nonché aver praticato una sorta di culto pagano della sua concubina, la divina Isotta (il tutto inquadrato, come possibile alternativa allo scetticismo, in un esibito ritorno a un platonismo precristiano).

${ }^{30}$ Quodque agitur per se, se nunquam deserit; ut quod / principium nunquam noverit ante suum. I Se movet bic per se nulloque agitante movetur; / coepit et ut nunquam sicque perennis erit. / Corporis ut moles animis est omnis adempta, / iuditio vivunt hi sine fine meo (Le poesie liriche di Basinio... op. cit: 20, vv. 35-40). Cf. Platone, Fedro, 245 c-e, 246 a-d. A Basinio peraltro tale pensiero giungeva attraverso la mediazione di Cicerone (Tusc. 1. 23, 53; De re pub. 6. 27) e Macrobio (Comm. in Somn. Scip. 2. 13, 1; 2. 15, 1-18), pressoché trasposti in versi dal parmense.

${ }^{31}$ Mensne eadem maneat tristi compage solutis, / ut reor, est paucis explicuisse datum. / Respondere canit curasque aequare Sichaeum / coniugis Aonio carmine Virgilius. / Ergo aliquid post fata sumus, vitaeque prioris, / quisquis habet, memores vivimus usque, locus. / Nam potare animas Lethaei oblivia fontis, / in formas demum quis putet ire novas? / Non mibi Pythagoras hoc unquam suaserit auctor, / sit licet in raris nomen in orbe suum. / Divinos animos caelesti ab sede profeto / communes brutis noluit esse Deus (Le poesie liriche di Basinio...... op. cit.: 20-21, vv. 41-52). 
si chiede se potrà mai ritrovarla $(3.10)^{32}$. Di fatto, il Poeta dell'Isottaeus svolge, nel corpo del racconto, il compito di consulente filosofico e spirituale che Basinio ricoprì storicamente nella corte di Sigismondo: il quale non mancò di suggellare la funzione sapienziale riconosciuta al suo poeta tumulandone le spoglie (a seguito della precoce morte intervenuta nel 1457) in una delle arche monumentali che ornano il fianco destro del suo Tempio Malatestiano, accanto a quelle che raccolgono i resti del famoso filosofo neoplatonico Gemisto Pletone, e di Giusto dei Conti, altro importante poeta (volgare), giunto per primo a Rimini a diffondere l'interesse per questa scuola di pensiero.

Se Basinio da Parma mise direttamente in scena il poeta nelle sue più alte attribuzioni (svolte col supporto sapienzale di antichi poeti e di filosofi), in quegli stessi anni a Ferrara due altri brillanti allievi di Guarino si trovarono invece a discuterne con opposti argomenti ${ }^{33}$. A lungo riluttante rispetto ai precetti del maestro, Tito Vespasiano Strozzi si dedicò liberamente lungo tutti gli anni '40 e '50 a una lirica capace di ridare freschezza alle topiche situazioni della poesia elegiaca, attraverso la contaminazione con stilemi del Canzoniere petrarchesco: nulla di sorprendente, dunque, se tale elegante disimpegno suscitò ben presto critiche nell'ambito della scuola guariniana. Famosa, in particolare, fu la reazione di un adolescente ungherese, Giano Pannonio (János Csemiczei), giunto tredicenne a Ferrara nel 1447, e destinato a diventare il più grande poeta dell'umanesimo magiaro, in quanto acuto cultore del genere epigrammatico e, soprattutto, intenso autore di elegie dedicate a profondi temi esistenziali. Di fronte a una lirica in cui lo Strozzi aveva rappresentato la propria gioia per un anello donatogli dall'amata ${ }^{34}$, in un testo non posteriore al 1450 Giano espresse il proprio fastidio con argomenti perfettamente conformi con la poetica guariniana:

\section{Cantatas alio transferre vetabere flammas, sic semper turpis te retinebit amor [...]. Excute damnosas captivo e pectore curas,}

${ }^{32}$ Ipse tamen referam sacri divina Platonis / plurima, quae Graiis me docuere sonis. / Ille putat rursus mortale in corpus ituras / esse animas pleno temporis orbe sui. / Nec minus Elysios post fata revisere campos / rursus et aeterna conditione trabi [...]. I Ipse Menoetiades, magno si crederis Homero, / venit Achilleos mortuus ante pedes / candidaque ossa sui Phtiae portaret ad urbem / fidus ut Aeacides iussit et inde fugit. I Sic te si affari cupiat tua cara puella, / forte potest vultus ante venire tuos. I Aeneaeque fuit simulacrum triste Creusae / ante oculos, trepidas dum fugit illa manus. / Quare ne dubites illam tibi posse reverti / et curas dulci voce levare tuas (Le poesie liriche di Basinio... op. cit.: 72-73, vv. 31-36, 43-52).

${ }^{33}$ Anche questo argomento ho trattato più distesamente in altre sedi: dapprima in Italo Pantani, «La fonte d'ogni eloquenza» op. Cit.: 278-282, più distesamente in Italo Pantani, Responsa poetae op. cit.: 113-127.

${ }^{34}$ Eroticon libri 1. 7 (Ad annulum quo donatus fuerat ab amica): cf. Della Guardia, Tito Vespasiano Strozzi... op. cit.: 18. 
deme Cupidineis et tua colla iugis;

prorsus et ingenuas convertere totus ad artes,

et grave neglecto carmen amore cane [...].

Scribere quae valeas sunt plurima praeter amorem,

sive nova arrident, sive vetusta placent;

bellica sive iuvat clarorum gesta virorum

carmine materiae conveniente suae,

sive referre togam ${ }^{35}$.

Questa elegia diede origine a una tenzone, destinata a grande fortuna (come provano memorie dello stesso Poliziano, su cui tornerò), che si protrasse per ben sei componimenti, durante i quali Tito Strozzi non difese la propria poesia (sulla scia del Marrasio) attribuendone la materia sentimentale all'azione di un sacro furore, quanto, al contrario, assumendo gli umili panni, petrarcheschi anche nelle immagini, dell'uomo consapevole del proprio errore, ma impossibilitato a liberarsene perché schiavo delle sue passioni:

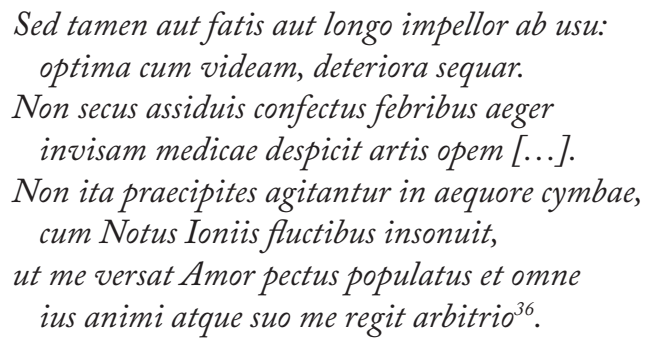

Naturalmente, in questa e in altre occasioni, lo Strozzi non mancò anche di celebrare la potenza di Amore e le sue azioni benefiche (compresa quella esercitata sui poeti); ma, in mancanza di una convinta adesione alla poetica platonica del furor, lo fece sostanzialmente in termini lucreziani e virgiliani. E infatti evidente, al contrario, come solo una piena attribuzione al poeta, su base platonica, di facoltà e compiti sapienziali e divinatori, poteva accreditare la sua arte a prescindere dai contenuti proposti, legittimando, in particolare, quelli amorosi.

${ }^{35}$ Elegia II 8, 79-80, 135-138, 163-168 (cf. Giano Pannonio, Poemata quae uspiam reperiri potuerunt omnia, 1784, ed. S. Teleki, 2 voll. Traiecti ad Rhenum (Utrecht), apud Barthol. Wild (rist. anast.: Budapest, Balassi Kiadó, 2002: 378-390).

36 Eroticon 1. 8. 55-58, 65-68 (cf. Della Guardia, Tito Vespasiano Strozzi... op. cit.: 18-33). Riscontri lessicali: per il v. 56 (Optima cum videam, deteriora sequar) cf. Ovidio, Met. 7. 20-21, s. Paolo, Rom. 7. 19, e Rvf 264. 136; per i vv. 57-58 (aeger / invisam medicae despicit artis opem) cf. Properzio 2. 4. 13, ma anche Rvf 75. 1-3; per i vv. 65-66 (agitantur in aequore cymbae) cf. Properzio 2. 4. 19-20 (in senso antifrastico), e Ovidio, Trist. 1. 1. 85, ma soprattutto Rvf 132 (10-12), 189, 235 e 272; per il v. 67 (me versat Amor, pectus populatus) cf. Ovidio, Am. 1. 2. 8. 
Il ruolo svolto dal pensiero di Marsilio Ficino in questa opera di conciliazione tra sapientia e furor, fin dall'epistola De divino furore a Peregrino Agli del 1457, è talmente noto da sconsigliare qui qualsiasi approfondimento ${ }^{37}$. Gioverà però ricordare che nel 1491, anno che si sarebbe rivelato ricchissimo di stimoli per Aires Barbosa (da pochi mesi giunto a Firenze), Ficino elaborò una seconda epistola De divino furore (quasi una postilla a quella del 1457), ora indirizzata a Pietro Dovizi, nella quale "i quattro furori platonici appaiono ancora uniti nel segno della poesia" ${ }^{38}$. Del resto, a prescindere da possibili influssi diretti sul nostro Barbosa, questi approdi dell'estetica ficiniana devono comunque interessarci in quanto fondamentali per le teorie poetiche di Angelo Poliziano, oggetto a sua volta di autentica venerazione da parte di Aires Barbosa, come provano i frequenti e commossi ricordi dell'Ambrogini nei suoi testi, pervasi dall'orgoglio di avere studiato presso un tale docente ${ }^{39}$.

Ricorderemo allora che, sempre in quel nodale 1491, Poliziano ripubblicò con nuova dedica i Nutricia, ossia la più pertinente a questo nostro percorso tra le Silvae: le prolusioni in versi con cui l'Ambrogini aveva aperto i suoi corsi degli anni '80, tutti incentrati su grandi poeti, da Omero a Virgilio a Stazio (laddove, proprio quando Barbosa giunse a seguirne le lezioni, egli si volse all'esegesi di Aristotele etico, fisico e soprattutto logico). Proprio dei Nutricia non si può qui omettere di ricordare, in particolare, alcuni imprescindibili versi:
Donec ab aetherio genitor pertesus Olympo socordes animos, longo marcentia somno
pectora, te nostrae, divina Poetica, menti
aurigam dominamque dedit [...].
Nam simul ac pulchro moderatrix unica rerum
suffulta eloquio dulcem sapientia cantum

${ }^{37} \mathrm{Cf}$. Marsilio Ficino, Lettere. I. Epistolarum familiarium liber I, a cura di S. Gentile. Firenze, Olschki, 1990: 19-28, epistola 1. 6. "Ficino riprende subito, a esordio di lettera, la dicotomia ars / furor proposta dal Bruni, attribuendo alla poesia del destinatario le positive caratteristiche di unopera divinamente ispirata. Ma la contrapposizione non è drastica ed è prospettata la possibilità di convivenza dei due elementi" (Coppini, "L'ispirazione per contagio...” op. cit.: 137).

${ }^{38}$ Ibid.: 141. Anche se "della poesia, e della persona, di Lorenzo; lomaggio al quale [...] condiziona il tono 'leggero' della trattazione” (ibid.).

${ }^{39}$ Celebrato in versi con appellativi quali meus Angelus, Gymnasii celebris celeber magister, poeta, orator; uomo capace di esprimersi Latino / cum sale, Cecropio cumque lepore simul; insomma, Ille, quasi e caelo sumptus, fuit angelus ignis (dall'epigramma In Nebulonem grammatistam iactuose uanum, ed. da José Henrique Rodrigues Manso, Comentário de Aires Barbosa ao segundo livro da Historia Apostolica de Arátor. Lisboa, Fundação Calouste Gulbenkian, 2011: 732-735, vv. 17-22, 27). Del resto, nel proprio commento ad Aratore (c. IIIr), Barbosa rivela non solo la conoscenza della Lamia (prolusione al corso sugli Analytica priora di Aristotele, recitata nel novembre del 1492), ma giunge a servirsi in funzione esegetica (c. cxxviv) del capolavoro filologico del Poliziano, i Miscellanea (cf. Manso, Comentário... op. cit.: 34, 408, 768). 
protulit, et refugas tantum sonus attigit aures,

[...] didicere quid usus

discrepet a recto; qui fons aut limes honesti;

qui ve fide cultus quid ius aequabile, quid mos, quid poscat decor et ratio [...].

Nunc age, qui tanto sacer bic furor incitet oestro

corda virum [...], expediam [...].

Nec tamen in nullis hominum simulachra refulgent

mentibus, arcanam caeli testantia Musam

permixtumque Iovem [...].

Is rapit evantem fervor [...],

inque suos humana ciet praecordia cantus.

[...] Mirantur et ipsi

saepe (quis hoc credat?) quae nuper cumque, recepto

numine, legitimi cecinere oracula vates ${ }^{40}$.

Poliziano teorizza dunque una poesia come dono di Dio agli umani, cui l'intelletto non bastava per uscire dalla barbarie. La sapientia divina (poi così cara a Barbosa), esprimendosi attraverso il canto dei poeti, diede avvio alla civiltà: e questo, naturalmente, fa della poesia la più alta delle scienze e delle arti, seconda solo alla teologia. Il sacro furore che ispira i poeti si deve alla particolare sensibilità di questi ultimi verso la divina armonia che regola il movimento dei cieli: quando tale furor li pervade, i poeti diventano come oracoli e vati, annunciatori di un messaggio di cui non sono proprietari. Si tratta di un quadro di pretta derivazione platonica e ficiniana (ancorché convergente con la poetica del subitus calor affermata da Stazio nelle sue Silvae), in cui la collocazione della poesia al vertice del sapere umano, in quanto rivelatrice di verità e di valori, giungeva da un lato a confermarne il ruolo fondativo nel quadro della cultura umanistica (senza possibilità di equivoco con l'interessata autopromozione di tanti verseggiatori), dall'altro a chiudere e sanare il lungo confronto che aveva contrapposto, nelle teorizzazioni d'età umanistica, la poesia ispirata da un incontrollabile furor, ma per questo estemporanea, inadeguata ai generi più nobili, rispetto a quella impegnata su contenuti alti, ma estranei all'esperienza e alla sensibilità dell'autore ${ }^{41}$.

\footnotetext{
${ }^{40}$ Poliziano, Silvae... op. cit.: 170-71, 176-80 (vv. 67-70, 75-77, 81-84, 139-140, 144, 156$158,163,169,182-184)$.

${ }^{41}$ Sulla "difficoltà latente", contenuta nel citato brano dei Nutricia, tra la concezione della poesia come "forma di raptus" e la natura dell'incivilimento umano, "che è una forma di persuasione prolungata nel tempo e resa istituzionale", si sofferma Paolo Paolini , "Sul tema dell'incivilimento attraverso la poesia nei 'Nutricia' del Poliziano e in altri autori”, in Italianistica, XII (1983): 217-234 (: 219); il quale, anche sulla base di altri testi polizianei, riconosce la soluzione dell'Ambrogini "in una mediazione che, pur salvando il primato ideale e cronologico della poesia, le attribuisse insieme una sorta di sapienza filosofica implicita e come aurorale,
} 
A tale risultato, peraltro, Poliziano poté giungere in virtù non solo delle premesse ficiniane, ma anche dell'attenzione da lui riservate alle elaborazioni del contesto ferrarese, e in particolare alle posizioni di Giano Pannonio, di cui molto apprezzò gli alti compiti conoscitivi attribuiti alla poesia. Lo testimoniano innanzitutto le ampie tracce lasciate, nell'elegia indirizzata dall'Ambrogini a Bartolomeo Della Fonte (1473), proprio dal carme di Giano a Tito Strozzi sopra ricordato (in particolare dai versi 183-206, dove l'autore enumera i prìncipi e gli Stati che Tito potrebbe celebrare), carme di cui peraltro perfino nelle Stanze (1. 51. 4: «teda legittima») si riecheggia la definizione elogiativa dell'amore coniugale (v. 81: «legitimas taedas»). Comprovano poi tale attenzione le memorie, riscontrabili nell'elegia in morte di Albiera degli Albizi (1473), di almeno cinque carmi del Pannonio (1.3. 9; 1. 6. 147$152 ; 1.9 .110 ; 1.10 .3 ; 1.12 .24)$; o ancor meglio l'intera praefatio alla selva Manto (1482), "esemplata fedelmente, nel suo impianto, nel suo svolgimento e nella sua trama allegorica, sull'analoga prefazione in distici che il Pannonio aveva premesso alla sua silva esametrica in onore di Guarino Veronese". Una definitiva conferma è infine rappresentata proprio dai Nutricia (1486), i cui primi sedici versi sono "chiaramente ricalcati su un analogo brano dell'elegia del Pannonio in morte della madre", già "ampiamente utilizzata nell'epicedio per Albiera degli Albizi" ${ }^{42}$.

Se l'ininterrotta fiducia barbosiana nell'arte poetica ebbe certo i suoi fondamenti in questi insegnamenti del Poliziano, è tuttavia indubbio che $\mathrm{i}$ criteri estetici adottati e promossi dall'autore dell'Antimoria (rifiuto di ogni retorica ambiguità, rinuncia a ornamenti mitologici, abbandono dei temi amorosi, decisa predilezione per quelli etico-religiosi, moderata apertura a quelli politici) mostrano forti punti in comune con altre componenti della cultura poetica fiorentina di fine ' $400^{43}$. Quest'ultima infatti, se da un lato non dimenticava l'alta funzione sapienziale e civilizzatrice attribuita alla poesia nel corso del secolo, dall'altro tendeva a darne un'interpretazione in chiave fortemente moralizzata, o meglio propriamente religiosa, aderendo in larga

destinata a successivi sviluppi in senso oratorio" (: 231).

${ }^{42}$ Tutti i riscontri e le citazioni relativi alla fruizione polizianea dei testi del Pannonio sono tratti da Francesco Bausi, "Poliziano e la poesia umanistica contemporanea", in V. Fera - M. Martelli (edd.), Agnolo Poliziano, poeta scrittore filologo... op. cit.: 165-193 (in particolare 176183, 190-191).

${ }^{43}$ Criteri estetici che si trovano ribaditi nelle espressioni di elogio riservate al poeta Aratore nella Praefatio al I libro della Historia apostolica: dove Barbosa difende, contro l'estremismo di taluni umanisti, lo stile semplice delle Sacre Scritture, e condanna, ricorrendo a una celebre immagine, prima petrarchesca (Famil. 23. 19) e poi polizianea (Ep. Paulo Cortesio suo) le "scimmie imitatrici" dell'antica eloquenza, specie se disposte ad accogliere espressioni empie. Viceversa, a suo giudizio "nostra id est sacra et ecclesiastica poesis tota casta est, sancta, virilis: in summa, virgo incorrupta" (cf. Barbosa, Aratoris cardinalis Historia apostolica... op. cit: cc. AırvIIIr, e Osorio, O Humanismo português... op. cit.: 54-55). 
misura agli insegnamenti di Girolamo Savonarola ${ }^{44}$. Non a caso, sempre a quel capitale (per la formazione barbosiana) 1491 risale l'Apologeticus de ratione poeticae artis, nel quale il seguitissimo frate definì poesia divinamente ispirata solo quella biblica, l'unica cui Dio avesse conferito sapientia e verità; bugiarda era dunque da ritenersi, in particolare, la poesia dei pagani (da non leggere né tantomeno imitare, soprattutto nell'uso dei miti), mentre quella moderna, pur se inferiore sul piano conoscitivo a teologia e filosofia, poteva essere accettata se evocatrice di virtù:

Verum quidem non amatoria, non laudes idolorum, non turpia, sed virorum fortium gesta atque moralia versibus descripserunt et bene usi sunt arte poetica et modo eius. Hos igitur damnare nec possum nec debeo ${ }^{45}$.

Quanto in profondità questo insegnamento incidesse nelle coscienze e nelle opere di numerosi letterati, è dato ben noto: si può qui ricordare il caso esemplare degli Epigrammata di Ugolino Verino, proprio nel 1491 sottoposti a rimozione dei contenuti mitologici e più distintamente neoplatonici, in componimenti peraltro fin dall'inizio dedicati a temi morali e teologici, nonché a elogi funebri di familiari e amici; ma analogo percorso è dato riscontrare in vari altri letterati di area medicea, come lo stesso Lorenzo, Giovanni Nesi o Girolamo Benivieni ${ }^{46}$. Minore successo, peraltro, ebbe l'opposizione del Savonarola alla poesia d'argomento religioso: condannata senz'altro dal frate come bestemmiatrice qualora "travestisse" mitologicamente la materia sacra, ma disapprovata anche in assenza di idolatre contaminazioni, in quanto le sue ambizioni retoriche ed estetiche avrebbero inevitabilmente distolto l'attenzione (di poeti e lettori) dalla verità dottrinale dei contenuti:

magis laudari de ornatu et carminum cursu ac vocabulorum integritate gestiunt quam de disciplina morum descripta. Nam et maiori eis curae est integritas locutionis quam doctrinae, nec possunt inanis gloriae vitare peccatum, immo nec abscondere ${ }^{47}$.

In realtà, perfino un fervente savonaroliano come il Verino, pur evitando la mitologia e perseguendo la massima sobrietà stilistica, non rinunciò affatto

${ }^{44}$ Riscontri tra la poetica di Barbosa e le tesi di Savonarola rilevò già José Sebastião da Silva Dias, A politica cultural da época de D. Joao III. Coimbra, Universidade de Coimbra, Instituto de Estudos Filosóficos, 1969: 222-225.

${ }^{45}$ Girolamo Savonarola, Apologeticus, in Id., Scritti fllosofici, a cura di G.C. Garfagnini e E. Garin. Roma, A. Belardetti, vol. 1, 1982: 211-272, in particolare 266.

${ }^{46} \mathrm{Su}$ questi temi cf. almeno Francesco Bausi, "Ugolino Verino, Savonarola e la poesia religiosa tra Quattro e Cinquecento", in G.C. Garfagnini (ed.), Studi savonaroliani. Verso il V centenario. Firenze, Sisnel, 1996: 127-135, e Ugolino Verino, Epigrammi, a cura di F. Bausi. Messina, Sicania, 1998: 33-123.

${ }^{47}$ Savonarola, Apologeticus... op. cit: 266. 
a praticare la poesia sacra (producendo ad esempio una versione in esametri dell'intero Vetus et Novum Testamentum), né a rivestirla di un moderato abbellimento classicheggiante; secondo un compromesso almeno in parte riconducibile all'opera conciliatrice di un altro predicatore, l'agostiniano Mariano da Genazzano, il quale divenne proprio in questi anni "il principale punto di riferimento di quanti (dal Poliziano al Brandolini, al Domizi) ritenevano possibile e legittimo sposare le verità cristiane con le attrattive della poesia e - in generale - con la cura della forma e dello stile, ossia, in una parola, con il magistero dei classici" ${ }^{4}$. Se dunque, analogamente, anche il nostro Barbosa, nel costante rifiuto di contenuti amorosi e abbellimenti mitologici, si sarebbe cimentato nella scrittura di un carme come l'Antimoria (ampiamente incentrato su contenuti biblici), di epigrammi a tema esclusivamente didattico, morale e civile, nonché nello studio di un poema, l'Historia apostolica di Aratore, esempio tra i più tipici di trasposizione poetica classicheggiante della sacra Scrittura, tali orientamenti estetici non potevano che nascere dal concorso delle suggestioni sopra riassunte: l'assoluta fiducia riposta dalla cultura umanistica nelle virtù conoscitive, formative e perfino sapienziali della poesia (come le aveva felicemente rappresentate Basinio, ed esemplarmente sintetizzate Poliziano); l'identificazione di tali virtù con quelle più strettamente concernenti l'ambito morale e civile, secondo l'approccio (per fermarci agli autori citati) di un Guarino e un Pannonio; l'impronta moralizzatrice apportata (anche sul piano delle risorse stilistiche, come l'abbandono della mitologia pagana) dalla predicazione savonaroliana, proprio negli anni in cui Barbosa soggiornava a Firenze; le aperture, infine, che anche la letteratura medicea più sensibile a tale insegnamento si riservava sul piano della forma e dell'imitazione dei classici, anche nel trattare argomenti sacri e spirituali.

${ }^{48}$ Francesco Bausi, “Ugolino Verino, Savonarola e la poesia religiosa...” op. cit.: 135. 